\title{
Common metabolic disorder (inborn errors of metabolism) concerns in primary care practice
}

\author{
Marisha Agana ${ }^{1}$, Julia Frueh ${ }^{1}$, Manmohan Kamboj $^{2}$, Dilip R. Patel ${ }^{1}$, Shibani Kanungo ${ }^{1}$ \\ ${ }^{1}$ Department of Pediatric and Adolescent Medicine, Western Michigan University Homer Stryker MD School of Medicine, Kalamazoo, MI, USA; \\ ${ }^{2}$ Department of Pediatrics, Nationwide Children's Hospital, Columbus, Ohio, USA \\ Contributions: (I) Conception and design: S Kanungo; (II) Administrative support: S Kanungo; (III) Provision of study materials or patients: S \\ Kanungo; (IV) Collection and assembly of data: M Agana, J Frueh, S Kanungo; (V) Data analysis and interpretation: S Kanungo; (VI) Manuscript \\ writing: All authors; (VII) Final approval of manuscript: All authors. \\ Correspondence to: Shibani Kanungo, MD, MPH. Department of Pediatric and Adolescent Medicine, Western Michigan University Homer Stryker \\ MD School of Medicine, 1000 Oakland Drive, Kalamazoo, MI 49008, USA. Email: Shibani.kanungo@med.wmich.edu.
}

\begin{abstract}
Inborn errors of metabolism (IEMs) are rare genetic or inherited disorders resulting from an enzyme defect in biochemical and metabolic pathways affecting proteins, fats, carbohydrates metabolism or impaired organelle function presenting as complicated medical conditions involving several human organ systems. They involve great complexity of the underlying pathophysiology, biochemical workup, and molecular analysis, and have complicated therapeutic options for management. Age of presentation can vary from infancy to adolescence with the more severe forms appearing in early childhood accompanied by significant morbidity and mortality. The understanding of these complex disorders requires special indepth training, American Board of Medical Genetics and Genomics (ABMGG) certification and experience. Most primary care physicians (PCPs) are reluctant to deal with IEM due to unfamiliarity and rarity of such conditions compounded by prompt progression to crisis situations along with paucity of time involved in dealing with such complex disorders. While there are biochemical geneticists aka metabolic specialists' expertise available, mostly in larger academic medical centers, with expertise to deal with these rare complex issues, their initial clinical presentation in most newborns, children, adolescents or adults including asymptomatic positive newborn screen (NBS), occur in the out-patient PCP settings. Therefore, it is important that PCPs' comfort to recognize early signs and symptoms is important to initiate appropriate diagnostic and therapeutic interventions, and be able to make appropriate referrals. The following article reviews common IEM clinical presentations for a robust diagnostic differential and discuss evaluation and management approaches of patients with known or suspected IEM.
\end{abstract}

Keywords: Inborn errors of metabolism (IEMs); biochemical genetics; primary care physician (PCP); acute illness protocol; D10W

Submitted Dec 17, 2018. Accepted for publication Dec 17, 2018.

doi: $10.21037 /$ atm.2018.12.34

View this article at: http://dx.doi.org/10.21037/atm.2018.12.34

\section{Introduction}

The term metabolism encompasses the net result of a multitude of complex biochemical processes that occur in living organisms to maintain cellular activities vital to sustain life (1). These processes are organized into specific metabolic pathways with the primary function of maintaining daily life activities. Each pathway depends on certain substrates and specific enzymes to ensure smooth functioning. Inborn errors of metabolism (IEMs) are a group of disorders which results from deficiency activity of a single enzyme in a metabolic pathway (2). Majority of the IEMs are inherited in an autosomal recessive manner. While individually they are rare, collectively they 
Table 1 PCP guidelines for considering IEM

In differential diagnosis of sepsis, anoxic encephalopathy or toxic ingestion

If symptoms persist even after treatment for suspected common conditions

If typical common laboratory tests fail to determine a definitive diagnosis

Can present either as acute illness or as chronic, recurrent or progressive disease at any age

Can occur even in the context of negative family history for genetic or metabolic disorder.

In cases of neonatal death from undetermined causes.

$\mathrm{PCP}$, primary care physician; IEM, inborn error of metabolism.

are common with an overall incidence of greater than 1:1,000 (3). With onset of universal expanded newborn screening (NBS), as noted in another article in this issues on NBS, the current data on IEMs detected by NBS is 1 in 3,234 (4).

\section{Classification}

IEMs can be categorized based on their onset, predominant signs and symptoms, main organs or systems affected, or acuity or chronicity of presentation (5). Disorders in category 1 usually involve a single organ or uniform presenting symptom, identified by specific laboratory tests and managed by specific organ system subspecialty practices like endocrinology, hematology, allergy/immunology and will not be discussed in this review.

Disorders in category 2 affecting specific biochemical or metabolic pathways in cytosol or organelle or intracellular transport or cofactors can be common to a large number of cells or organs presenting with more variable symptoms and co-morbid conditions that include multiple organ systems. These disorders can be classified into three sub-groups including: (I) intoxication due to defects in the intermediary metabolic pathway resulting in the accumulation of toxic compounds proximal to the metabolic block [example: urea cycle defect (UCD), amino acid disorders]; (II) deficiency in energy production or utilization [example: mitochondrial disorders, glycogen metabolism disorders, fatty acid oxidation disorders (FAO)]; and (III) complex molecules involving organelles (example: lysosomes, peroxisomes, Golgi and endoplasmic reticulum) and cofactors (6).

\section{Common IEM presentations}

Children and adolescents with IEMs have a wide spectrum of clinical presentations from appearing physically normal to having distinctive dysmorphic physical features. While majority of them appear physically normal at birth, many can present with significant non-specific signs and symptoms common to other serious medical conditions (7). It is imperative to keep a high index of suspicion in differential diagnosis for prompt IEM identification as the institution of appropriate therapy, preventive measures and compliance helps avoid severe morbidity or even mortality in some cases.

In the antenatal period, true irreversible major malformations due to IEMs can be found in O-glycosylation disorder; in cholesterol synthesis defects, in amino acid synthesis disorders (glutamine and asparagine) and in mitochondrial disorders $(8,9)$.

\section{Newborn/infancy period (<12 months)}

Examination of a newborn for subtle signs of IEMs starts from a cursory examination of the infant from head to toe. It may be practical first to examine areas that require auscultation while the infant is quiet as he or she may be somewhat irritable in early transition and undressing the infant or moving him or her around may be disruptive. In a seemingly healthy newborn most of the information is structural involving the hair, skin, skeleton or all three that may anticipate future problems. Examination findings usually relate to major organ dysfunction or failure, most commonly hepatic, neurologic or immunologic and less commonly cardiac or pulmonary. While it is apparent that some IEMs present with characteristic dysmorphic features, majority of the newborns with IEM appear otherwise normal but clinically sick presenting with overwhelming septicemia or failure to thrive (see Table 1).

\section{Dermatologic}

Absence of hair or alopecia can signal defects in mineral and vitamin metabolism such as acrodermatitis enteropathica (a disorder in zinc metabolism), biotin (vitamin B) responsive multiple carboxylase deficiency (MCD), calciferol (vitamin D) metabolism defects; nutritional deficit related fatty acid deficiency as well as "classical" organic aciduria/acidemia.

Brittle hair can be seen in copper metabolism disorders, argininosuccinic aciduria or citrullinemia as well as 
mucopolysaccharidosis.

Ichthyosis or dry, thickened scaly skin can be seen in lysosomal storage disorders (LSD), complex phospholipids and fatty acid synthesis/remodeling defects, cholesterol synthesis defects, peroxisomal disorders; dolichol synthesis and recycling defects and other lipid metabolism disorders.

The presence of vesicular or bullous lesions can suggest acrodermatitis enteropathica, biotinidase deficiency, holocarboxylase synthetase deficiency, lipin 2 deficiency, zinc deficiency, classical organic acidurias/acidemias.

Cutis laxa syndrome is comprised of diseases characterized by wrinkled, redundant, inelastic and sagging skin secondary to defective synthesis of elastic fibers and other proteins of the extracellular matrix and several metabolic disorders have been found to be associated with this including copper metabolism defects, combined disorder of $\mathrm{N}$ - and O-linked glycosylation CDG defects and proline synthesis defects.

Presence of xanthomas (yellowish cholesterol-rich deposits) points to disorders with cholesterol metabolism while lipomatosis is indicative of triglyceride and phospholipid synthesis defects.

\section{Ophthalmologic}

Routine use of the ophthalmoscope and ophthalmologic examination can provide vital findings suggestive of IEM. Cataracts can be suggestive of galactosemias, galactokinase deficiency, peroxisomal biogenesis defects, sorbitol dehydrogenase deficiency.

Corneal clouding is seen in tyrosinemia type II, cystinosis, mucopolysaccharidoses (MPS) type I syndromes such as Hurler and MPS VI or Maroteaux-Lamy syndromes. Dislocation of the lens should raise suspicion for classic homocystinuria, sulfite oxidase deficiency, Marfan's and Marchesani syndromes.

The presence of keratitis with corneal opacities can be seen in tyrosinemia type II and Fabry disease while microcornea can be manifested in Ehlers Danlos type IV. Macular colobomata can be evident in familial hypomagnesemia. Conjunctivitis and blepharitis can be apparent in cystinosis, tyrosinemia type II, propionic acidemia (PA), and MCD.

\section{Cardiac}

Cardiac failure secondary to dilated cardiomyopathies are rare but can be seen in neonates with long branched chain FAO disorders, mitochondrial disorders and Pompe's disease. When neonates present with cardiac arrhythmias, consider IEMs.

\section{Hepatic}

In the examination of the abdomen, the presence of organomegaly (liver, spleen and kidney) associated with jaundice and findings of liver damage can be seen in the form of: storage (glycogen, neutral lipids, complex lipids); cholestasis, fibrosis/cirrhosis and inflammatory and immune changes. A firm or rock-hard consistency of the liver indicates tyrosinemia type I, galactosemia, GSD type IV, alpha 1 antitrypsin deficiency, Wilson disease, cystic fibrosis, Niemann Pick or Gaucher disease. Normal or soft liver consistency associated with splenomegaly strongly suggests LSD.

\section{Musculoskeletal}

In the examination of the musculoskeletal system, many IEMs can present with severe hypotonia, muscle weakness and poor muscle mass can be seen in urea cycle disorders (UCD) and organic acidemias. Severe neonatal generalized hypotonia and progressive myopathy can be seen in primary and secondary mitochondrial disorders, fatty acid oxidation defects (FAO), peroxisome biogenesis disorders (PBD), glycogen storage diseases (GSD), and some other lysosomal storage diseases (10).

\section{Neurologic}

The most common manifestations of IEM in the neonatal period are decreased level of consciousness; seizures; hypo- or hypertonia. Coma in a neonate is likely due to an intoxication metabolic disorder such as UCD and OA and often treatable. Hypotonia as the only presentation in a neonate caused by an IEM is rarely treatable $(5,6)$.

Mental status changes in a newborn can be seen in several underlying common biochemical derangements, such as metabolic acidosis, hyperammonemia, or hypoglycemia (7). The alteration in mentation may progress from irritability to lethargy and ultimately a comatose state. In general, coma due to metabolic derangements is associated with abnormal movements of the limbs or elevated muscle tone, or neuro-vegetative symptoms such as hiccups. A comatose state associated with hypotonia is more commonly caused by non-metabolic processes but may also be seen in IEM such as UCDs (2,5-7). The exact onset and temporal course of altered mental status in a neonate may help differentiate between two major groups of underlying disease mechanisms in IEMs: if an initially healthy neonate starts to rapidly deteriorate after an initially asymptomatic 
Table 2 Distinctive facial abnormalities

\begin{tabular}{ll}
\hline IEM & Facial features \\
\hline Zellweger syndrome (absence of peroxisomes) & $\begin{array}{l}\text { Large fontanelle, prominent forehead, flattened nasal bridge, epicanthal folds and } \\
\text { hypoplastic supraorbital ridge } \\
\text { Epicanthal folds, flattened nasal bridge, petite nose with anteverted alae nasi, long } \\
\text { philtrum }\end{array}$ \\
Glutaric aciduria type II & $\begin{array}{l}\text { Macrocephaly, high forehead, flattened nasal bridge, short anteverted nose; genitourinary } \\
\text { abnormality of hypospadias in males, aural anomalies and rocker bottom feet }\end{array}$ \\
$\begin{array}{l}\text { Smith-Lemli-Opitz syndrome (cholesterol } \\
\text { biosynthetic defect) }\end{array}$ & $\begin{array}{l}\text { Epicanthal folds, flat nasal bridge, syndactyly, cataracts and genital abnormalities } \\
\text { Congenital glycosylation disorders }\end{array}$ \\
$\begin{array}{l}\text { Inverted nipples and lipodystrophy } \\
\text { Mucopolysaccharidoses }\end{array}$ & Coarse facies \\
\hline
\end{tabular}

IEM, inborn error of metabolism.

interval (hours to weeks), this may indicate that intoxication due to an IEM is the underlying cause. On the other hand, IEM that lead to energy deficiency may show a progressively worsening clinical course without initial symptom-free interval and with more variable severity of initial presentation. These IEM are also more likely to be associated with cardiac or hepatic abnormalities.

Seizures in the neonatal period are another common initial finding of IEM, and unexplained or intractable seizures in a neonate should always raise the suspicion for IEM (11). Seizures may present with a mixed semiology including partial, generalized, myoclonic, and tonic seizures. Classic EEG patterns, such as a burst-suppression pattern, may be present. Many treatable IEM however present with isolated, generalized seizures, such as pyridoxine and folinic acid responsive seizures (6).

Neonatal hypotonia as a major presenting symptom of an IEM is rare, but may occur in rarely treatable mitochondrial or neurotransmitter defects. However, there are a few treatable metabolic disorders presenting with severe generalized hypotonia which can mimic neuromuscular disorders, such as FAO. Hypotonia in IEM is usually associated with other symptoms.

\section{Children and adolescents}

Some IEMs may not manifest in the neonatal period or until later in infancy or early childhood or even adolescence with asymptomatic duration but often present acute and rapid clinical deterioration. However, some patients can develop distinctive facial abnormalities (see Table 2) or have specific odors (see Table 3) raising a high level of suspicion for IEMs.

\section{Cardiac manifestation}

Some IEMs may present with predominantly cardiac manifestation of heart failure due to dilated hypertrophic cardiomyopathy. These children also suffer failure to thrive and can be found to have FAO, respiratory chain disorders or Pompe disease (10).

\section{Hepatic and gastrointestinal manifestations}

There are six main clinical groups that can be categorized as: (I) hepatomegaly with hypoglycemia without liver failure is suggestive of glycogenesis type 1 or III or gluconeogenesis defects. (II) Liver failure and hypoglycemia are seen in fructosemia, galactosemia, tyrosinemia type I as well as respiratory chain disorders. (III) Cholestatic jaundice with failure to thrive can be found in alpha-1anti-trypsin deficiency, bile acid metabolic disorders, peroxisomal disorders, Niemann-Pick type C disease, congenital disorder of glycosylation (CDG), citrine deficiency and mitochondrial DNA depletion. (IV) Hepatic steatosis is suggestive of FAO as well as UCDs. (V) Hepatosplenomegaly can be suggestive or storage disorders manifested in lysosomal diseases. (VI) Diarrheal disorders secondary to malabsorption may be caused by disaccharidase deficiency and triglyceride synthetic disorders (5).

\section{CNS manifestations}

From infancy in adolescence, IEM may present with recurrent episodes of acute neurological dysfunction, or 
Table 3 Different odors suggesting IEM

\begin{tabular}{ll}
\hline IEM & Abnormal odor \\
\hline Maple syrup urine disease (MSUD) & Maple syrup \\
$\begin{array}{l}\text { Isovaleric acidemia or glutaric } \\
\text { acidemia type II }\end{array}$ & Sweaty feet \\
Cystinuria and tyrosinemia type I & Sulfur \\
Tyrosinemia type I & Boiled cabbage \\
Trimethylaminuria or dimethylglycine & Old fishy \\
dehydrogenase deficiency & \\
Multiple carboxylase deficiency (MCD) & Cat's urine \\
Phenylketonuria & Mousy \\
\hline
\end{tabular}

IEM, inborn error of metabolism.

as chronically progressive neurologic disability. Symptom onset may occur at any age between early infancy and as late as adulthood (5).

Some IEM present with acute, episodic neurological dysfunction. In those IEM, specific triggers may be identified, such as prolonged fasting or change in physical activity level. Examples for IEM that present with recurrent attacks are defects in gluconeogenesis and maple syrup urine disease, among many others. Recurrent strokes or strokelike episodes at a young age can be seen in homocystinuria, mitochondrial disorders such as MELAS and UCDs.

Recurrent episodes of ataxia may be seen in late-onset maple syrup urine disease or pyruvate dehydrogenase deficiency.

Acute psychiatric symptoms may be evident in some IEMs. These are rather difficult to identify, especially if concomitant extra-neurological symptoms or laboratory abnormalities are mild but symptoms range from psychosis to anxiety or aggression (12). Examples of IEM manifesting with acute psychiatric symptoms are the porphyrias (may also present with acute peripheral neuropathy), Wilson disease, and late-onset UCDs $(12,13)$.

On the other hand, IEM may manifest as a progressive decline in neurocognitive functioning, arrest or regression in development or intellectual disability. These usually occur in conjunction with other neurological abnormalities. Isolated developmental delay or regression as well as isolated intellectual disability without other neurological signs is less commonly caused by a metabolic disorder (9,14-16). IEM that commonly cause intellectual disability are phenylketonuria, homocystinuria or Smith Lemli Opitz syndrome. Developmental regression may be associated with a wide range of neurological findings. Extrapyramidal symptoms for example may occur in Lesch-Nyhan syndrome or Pelizaeus Merzbacher syndrome. Opisthotonus may be seen in Krabbe or Gaucher disease. Another concomitant finding suggestive of specific diagnoses is progressive paraplegia or spasticity, which is classically seen in metachromatic leukodystrophy. Some patients with an underlying IEM may have been falsely diagnosed with cerebral palsy. Progressive neurodegeneration with ataxia can be seen in ataxia-teleangiectasia. During the neonatal period, seizures are a common manifestation of IEM at any age, as seen in Niemann-Pick or Gaucher disease.

Seizures are a common finding in IEM not only in the neonatal period but at any age (17). Factors raising concern for an underlying IEM are a positive family history, presence of other neurological abnormalities, unexplained background slowing on EEG, or worsening with certain anti-epileptic drugs or dietary changes

Infants and children who present with seizures fall into the category of defects in glycine, serine metabolism and cofactor disorders. Intractable seizures can also be manifestations of vitamin metabolism disorders aka pyridoxine (vitamin B6) and biotin (vitamin B7) (18).

Severe hypotonia are observed in congenital hyperlactatemias, mitochondrial disorders, UCD, nonketotic hyperglycinemia, sulfite oxidase deficiency and peroxisomal disorders. These so-called metabolic myopathies include most FAO disorders and carnitine shuttle defects (10).

Finally, IEMs involved with complex molecules such as peroxisomal diseases are associated with dysmorphologies, seizures, severe hypotonia and cholestatic liver disease while lysosomal disorders represent a group that involve abnormal accumulation of metabolites in various organs and tissues resulting in multi-system dysfunction: dwarfism, hepatosplenomegaly, recurrent infections, skeletal dysplasias and neurocognitive impairment (8).

\section{Management approach}

Infants, children and adolescents with IEM who appear normal may succumb to life-threatening conditions. Appropriate acute illness protocol (see Table 4) and specific supportive therapies are needed to assure the patient's survival. Many patients will require respiratory and circulatory support. Most will require rehydration, correction of electrolyte imbalance and even treatment of overwhelming infection from opportunistic organisms that 
Table 4 IEM acute illness protocol template for PCP

\begin{tabular}{|c|c|}
\hline Variable & Definition \\
\hline IEM disorders & $\begin{array}{l}\text { OA, mitochondrial disorders, GSD, } \\
\text { UCD }\end{array}$ \\
\hline Common illness/conditions & $\begin{array}{l}\text { Viral/bacterial infections; surgical } \\
\text { procedures }\end{array}$ \\
\hline Medical attention (PCP, ER) & STAT \\
\hline STAT laboratory tests & $\begin{array}{l}\text { BMP, urinalysis (+/-). CBC, NH3 } \\
\text { (ammonia) }\end{array}$ \\
\hline STAT IV access & $\begin{array}{l}\text { D10 W + } 0.5 \text { normal saline at } \\
1.5 \text { times maintenance (per age } \\
\text { guidelines) }\end{array}$ \\
\hline Feeding & $\begin{array}{l}\text { NPO with above IV fluids until } \\
\text { hemodynamically stable }\end{array}$ \\
\hline Call & $\begin{array}{l}\text { Local friendly biochemical/IEM } \\
\text { specialist, intensive care unit }\end{array}$ \\
\hline
\end{tabular}

$\mathrm{PCP}$, primary care physician; IEM, inborn error of metabolism; $\mathrm{OA}$, organic aiduria; GSD, glycogen storage disease; UCD, urea cycle disorders; BMP, basic metabolic panel; CBC, complete blood count; D10W, dextrose solution containing $10 \%$ glucose.

uncorrected can lead to persistent catabolic state and failure of definitive therapeutic intervention (19).

\section{Nutrition}

Whatever the IEM disease condition, addressing the nutritional requirements of the patient is very important. Most patients that are hemodynamically unstable or septic may have to have nothing by mouth (NPO) initially for first day or two to temporarily eliminate exposure to or reduce the incidence of toxic metabolites in the system. Total parenteral nutrition (TPN) is the preferred choice in those cases wherein effective enteral nutrition is not acceptable due to intestinal intolerance, high energy or high glucose requirements or the introduction of invasive techniques are needed for immediate detoxification. If the patient is stable, enteral or oral nutrition is encouraged utilizing one of these four types of diet: normal, lowprotein, carbohydrate-restricted, high-glucose with or without lipid restriction.

\section{Hydration}

Hemodynamic stability of a sick neonate, infant or child entails general resuscitative measures by providing intravenous fluid administration of $10 \%$ dextrose solution (D10W) containing $75 \mathrm{mmol} / \mathrm{L}$ of sodium $(0.5 \mathrm{NS})$ +/- $20 \mathrm{mmol} / \mathrm{L}$ of potassium may be started to reduce the catabolic state and reduce exposure to the offending nutrients. Fluid replacement must occur gradually over the first 48 hours with careful monitoring to avoid cardiac overload. Correction of metabolic acidosis and electrolyte imbalance as well as promotion of anabolic state must be performed gradually over the subsequent days based on the specific therapeutic requirements of the IEM disease condition.

\section{Toxin removal}

Those patients with acute metabolic toxicity such as UCD or branched chain organic acidurias (BCOAs) may require extracorporeal measures to remove the toxins circulating in the bloodstream especially if the affected patient is comatose or semi-comatose. These procedures may be in the form of continuous veno-venous hemodiafiltration (CVVHDF) or hemodialysis (HD) and peritoneal dialysis (PD).

\section{CNS deterioration}

Infants and children with IEM can suffer metabolic encephalopathy and these include: MSUD, BCOAs, and UCD. These patients can develop overhydration, cerebral edema and acute protein malnutrition if not properly managed. Careful monitoring of blood glucose, lactate, calcium, ammonia levels are imperative to know when to implement correction of the electrolyte and acid-base imbalance. Specific therapies such as insulin administration may be used to suppress severe catabolism or infusion of massive doses of specific vitamins may be essential for vitamin-dependent disorders (14).

In the presence of intractable seizures, vitamin responsive IEM must be considered. Giving certain vitamins like pyridoxine, biotin, thiamine, folinic acid and vitamin $\mathrm{K}$ may be life-saving and warranted to alleviate some of the symptoms.

\section{Liver failure}

Many IEM conditions present with jaundice, coagulopathy, hepatocellular necrosis with elevated levels of serum transaminases, hypoglycemia, ascites and generalized edema 
resulting in liver failure. These disorders include: fructosemia, galactosemia, tyrosinemia type 1, neonatal hemochromatosis, respiratory chain disorders and transaldolase deficiency. These abnormalities are often associated with mellituria, hyperammonemia, hyperlactatemia, hypoglycemia, hypertyrosinemia and hypermethioninemia secondary to advance hepatocellular disease.

\section{Cardiac failure}

Heart failure and dilated hypertrophic cardiomyopathy may be the presenting symptom of the metabolic disorders associated with FAO disorders, mitochondrial disorders or Pompe disease. These patients demonstrate hypotonia, muscle weakness and failure to thrive. IEMs involving long chain FAO can also manifest arrhythmias and conduction defects that may lead to cardiac arrest and death.

\section{Management of recurring attacks and chronic problems}

Almost half of patients with IEM may present with delayed onset of symptoms. The symptom-free period may last for months or even years extending into adolescence or adulthood. These periods of normalcy may be punctuated with acute episodes of attacks often precipitated by an intercurrent event related to protein intake, prolonged fasting, prolonged exercise or any condition that exacerbates protein catabolism. It is therefore imperative to coordinate care with the metabolic specialist to anticipate these crisis situations when they do occur.

\section{Conclusions}

The clinical outcome of children with IEM depends on multiple factors. These include type of the disorder, severity of the underlying metabolic defect, ability to make the diagnosis early, availability of specific adequate treatment options and appropriate institution of the definitive therapeutic intervention. Depending on all these variables, some IEMs have relatively better prognosis than others. Many of these children are able to sustain longer lives but many may be at risk for developing progressive neurologic deficits, learning disabilities and mental retardation. It is therefore important for primary care providers to know how to recognize those conditions, manage them in the interim while awaiting definitive diagnosis and refer them to the appropriate metabolic specialist for collaborative management of these patients.

\section{Acknowledgements}

None.

\section{Footnote}

Conflicts of Interest: The authors have no conflicts of interest to declare.

\section{References}

1. Kambhoj M. Inborn Errors of Metabolism. In: Neurodevelopmental Disabilities: Clinical Care for Children and Young Adults - Google Books. Springer, 2011:53-68.

2. El-Hattab AW. Inborn Errors of Metabolism. Clin Perinatol 2015;42:413-39.

3. Campeau PM, Scriver CR, Mitchell JJ. A 25-year longitudinal analysis of treatment efficacy in inborn errors of metabolism. Mol Genet Metab 2008;95:11-6.

4. CDC Grand Rounds: Newborn Screening and Improved Outcomes. Cited 2018 Dec 12. Available online: https:// www.cdc.gov/mmwr/preview/mmwrhtml/mm6121a2.htm

5. Saudubray JM, Garcia-Cazorla À. Inborn Errors of Metabolism Overview: Pathophysiology, Manifestations, Evaluation, and Management. Pediatr Clin North Am 2018;65:179-208.

6. Saudubray JM. Clinical Approach to Inborn Errors of Metabolism in Paediatrics. In: Inborn Metabolic Diseases. Berlin, Heidelberg: Springer Berlin Heidelberg, 2012:3-54.

7. Kölker S, Cazorla AG, Valayannopoulos V, et al. The phenotypic spectrum of organic acidurias and urea cycle disorders. Part 1: the initial presentation. J Inherit Metab Dis 2015;38:1041-57.

8. Bellettato CM, Hubert L, Scarpa M, et al. Inborn Errors of Metabolism Involving Complex Molecules. Pediatr Clin North Am 2018;65:353-73.

9. Kanungo S, Soares N, He M, et al. Sterol metabolism disorders and neurodevelopment-an update. Dev Disabil Res Rev 2013;17:197-210.

10. El-Gharbawy A, Vockley J. Inborn Errors of Metabolism with Myopathy. Pediatr Clin North Am 2018;65:317-35.

11. Sharma S, Prasad A. Inborn Errors of Metabolism and Epilepsy: Current Understanding, Diagnosis, and Treatment Approaches. Int J Mol Sci 2017;18:1384.

12. Nia S. Psychiatric signs and symptoms in treatable inborn errors of metabolism. J Neurol 2014;261:S559-68. 
13. Sedel F. Inborn Errors of Metabolism in Adults: A Diagnostic Approach to Neurological and Psychiatric Presentations. In: Inborn Metabolic Diseases. Berlin, Heidelberg: Springer Berlin Heidelberg, 2012:55-74.

14. van Karnebeek CDM, Bowden K, Berry-Kravis E. Treatment of Neurogenetic Developmental Conditions: From 2016 into the Future. Pediatr Neurol 2016;65:1-13.

15. Mochel F, Sedel F. Inborn Errors of Metabolism in Adults: A Diagnostic Approach to Neurological and Psychiatric Presentations. In: Inborn Metabolic Diseases. Berlin, Heidelberg: Springer Berlin Heidelberg, 2016:71-89.

16. Sklirou E, Lichter-Konecki U. Inborn Errors of

Cite this article as: Agana M, Frueh J, Kamboj M, Patel DR, Kanungo S. Common metabolic disorder (inborn errors of metabolism) concerns in primary care practice. Ann Transl Med 2018;6(24):469. doi: 10.21037/atm.2018.12.34
Metabolism with Cognitive Impairment. Pediatr Clin North Am 2018;65:267-77.

17. Saudubray JM, Garcia-Cazorla A. Clinical Approach to Inborn Errors of Metabolism in Pediatrics. In: Inborn Metabolic Diseases. Berlin, Heidelberg: Springer Berlin Heidelberg, 2016:3-70.

18. Dionisi-Vici C, Ogier de Baulny H. Emergency Treatments. In: Inborn Metabolic Diseases. Berlin, Heidelberg: Springer Berlin Heidelberg, 2012:103-11.

19. Schiff M, Mochel F, Dionisi-Vici C. Emergency Treatments. In: Inborn Metabolic Diseases. Berlin, Heidelberg: Springer Berlin Heidelberg, 2016:109-17. 